\title{
Editorial
}

\section{Green Transportation System and Safety}

\author{
Wuhong Wang, ${ }^{1}$ Geert Wets, ${ }^{2}$ and Heiner Bubb ${ }^{3}$ \\ ${ }^{1}$ Department of Transportation Engineering, Beijing Institute of Technology, Beijing 100081, China \\ ${ }^{2}$ Transportation Research Institute (IMOB), Hasselt University, 3590 Diepenbeek, Belgium \\ ${ }^{3}$ Lehrstuhl für Ergonomie, Technische Universität München, 85747 Munich, Germany
}

Correspondence should be addressed to Wuhong Wang; wangwuhong@bit.edu.cn

Received 1 June 2014; Accepted 1 June 2014; Published 16 June 2014

Copyright (C) 2014 Wuhong Wang et al. This is an open access article distributed under the Creative Commons Attribution License, which permits unrestricted use, distribution, and reproduction in any medium, provided the original work is properly cited.

Effectiveness and efficiency have been accepted as the measures for the transportation system as well as its components performance. However, highlighted by the concept of sustainability, environmental impacts of the transportation system must be standardized for traffic participants, roads, and infrastructure from planning to operation. With consideration of the road transport accounting for $75 \%$ of the world's total carbon dioxide emissions from fossil fuel combustion and the transportation systems being responsible for more than $20 \%$ of world energy consumption, statistical results raise the following problems: what efforts should we make to solve such environmental problems and what countermeasures should we conduct to balance the energy saving and the demand of mobility? Within the sustainable approaches, green traffic and green transportation system are now widely discussed.

Green vehicles, smart road, C-2-X communications for green intelligent transportation systems control, and green urban traffic are proved to have considerable effectiveness. Green vehicles, with new alternative energy as their key feature, are intended to be more environmentally friendly than conventional internal combustion engine vehicles running on gasoline or diesel. Electricity, hybrid electricity, compressed air/natural gas, biofuel, solar power, and so forth can be used as the embodied energy of the vehicles which represent the different types of green vehicles. The development of green vehicles triggers the challenges not only in the manufacture, but also in the use of vehicles and the service provided by traffic system.

Smart road; C-2-X communications including communications between/among vehicles, roadways, roadside, backend infrastructure, and so forth; and the assistance systems based on driver-vehicle unit lay the foundation of the traffic information network and enable a smooth, efficient, and accident-free traffic. The ongoing information system in ITS and driver assistance systems will provide guidance to the road users and, moreover, contribute to the emission control and safety.

The overbuilt cities experience the consequence of overloaded traffic demand and the worsening environment. Walking and cycling traffic are encouraged by enhancing the role of public transport in order to control $\mathrm{CO}_{2}$, air pollution, and noise. Focusing on the improvement of traffic conditions, productivity of cities will benefit from green urban traffic by mitigating the traffic congestions and delay. Furthermore, user-oriented urban traffic will contribute to the safety of vulnerable road users.

It is believed that the green transportation system (GTSS) will be a prime target for obtaining eco-friendly transportation systems and safety.

Wuhong Wang

Geert Wets

Heiner Bubb 


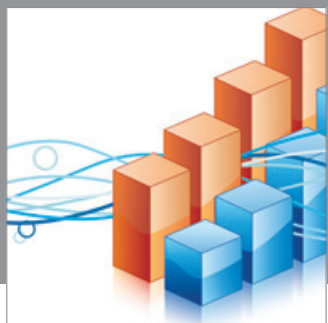

Advances in

Operations Research

mansans

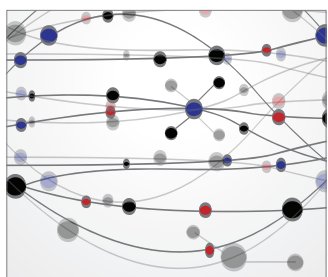

The Scientific World Journal
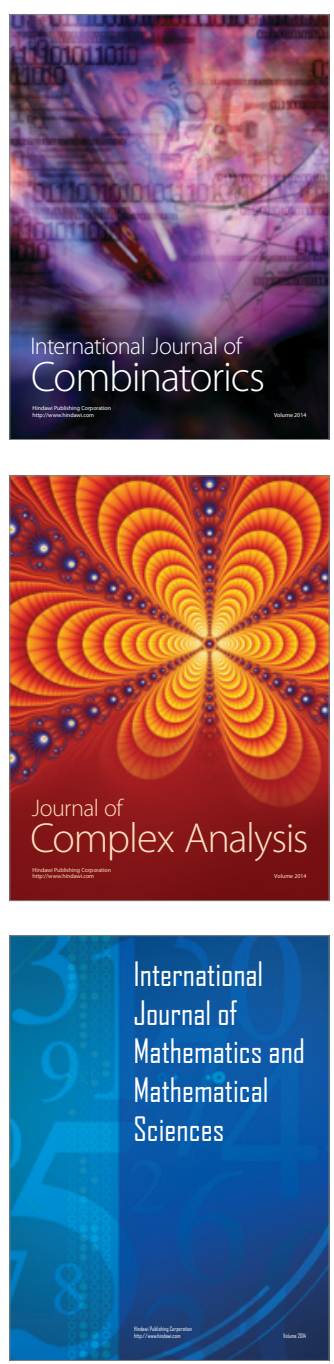
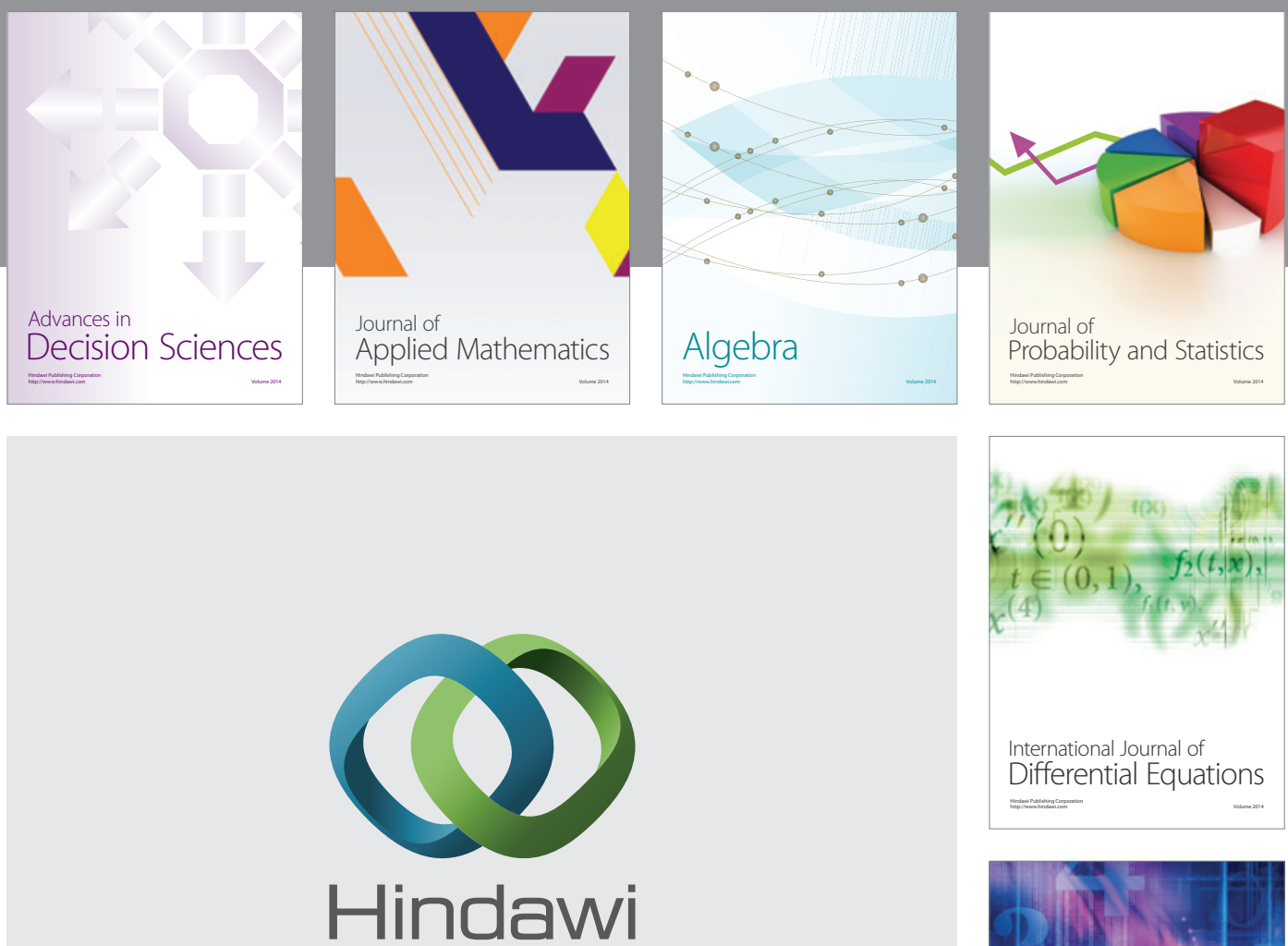

Submit your manuscripts at http://www.hindawi.com
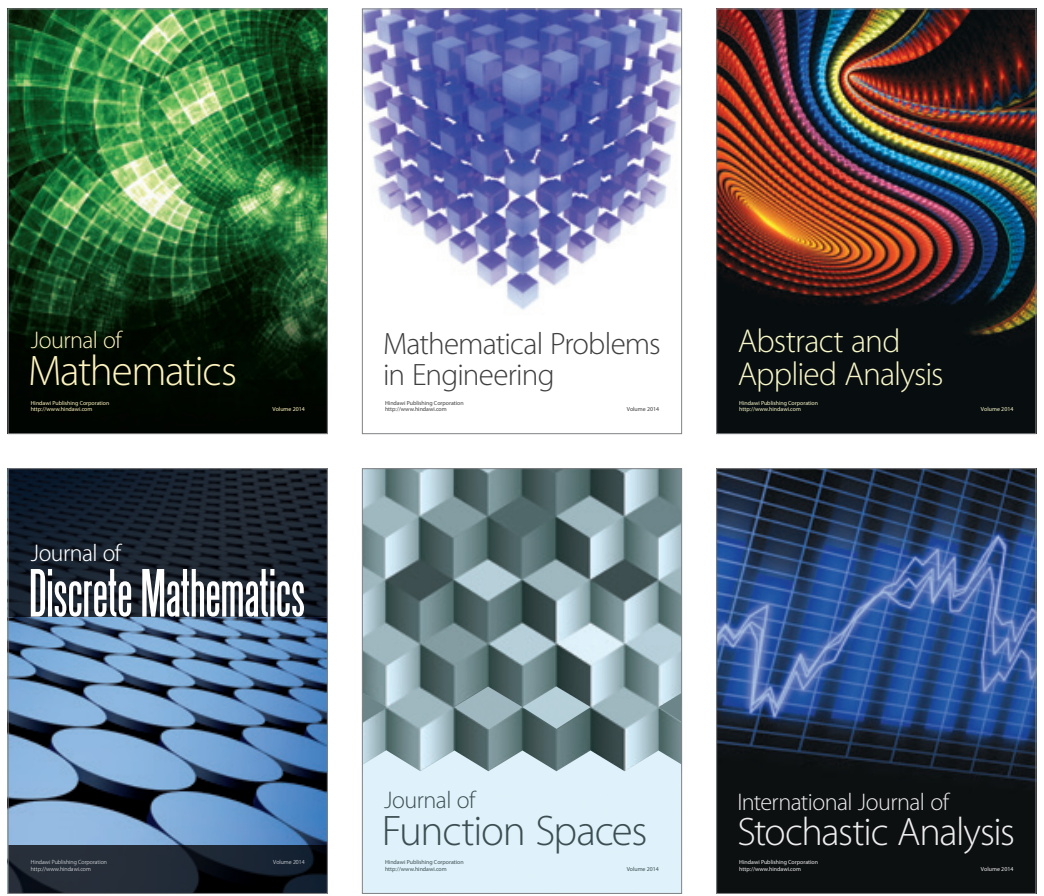

Journal of

Function Spaces

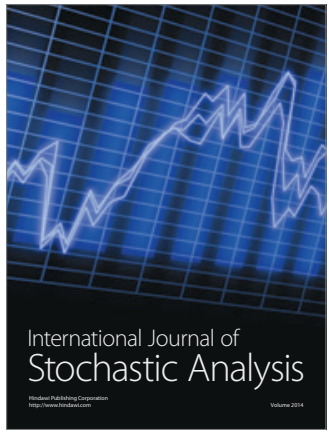

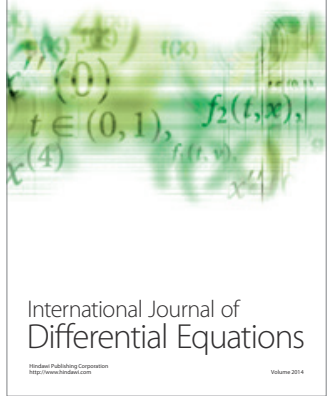
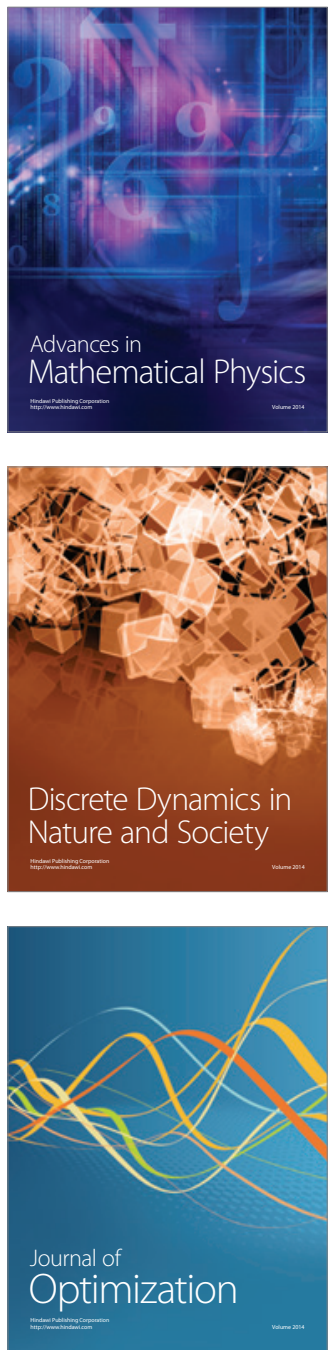